\title{
La participación de las familias en la escuela: una cuestión compleja
}

\section{Families involvement in school: a complex question}

Núm. 7 (2016), pp. 28-47

Andrés Cabello, Sergio*1

Giró Miranda, Joaquín ${ }_{2}$

Recibido: abril, 2016 Aceptado: septiembre, 2016

JEL Clasif: I2O, I24, I28

DOI: $10.5944 /$ reppp.7.2016.16302

\footnotetext{
*1 Sergio Andrés Cabello. Doctor en Ciencias Políticas y Sociología por la Universidad del País Vasco, especializado en identidades colectivas, Sociología de la Educación y Sociedad y Estructura Social. Universidad de La Rioja. E-mail: sergio.andres@unirioja.es. Orcid: http://orcid.org/oooo-0002-1703-005X

*2 Joaquín Giró Miranda. Profesor Titular de Sociología de la Universidad de La Rioja, Doctor por la Universidad de La Rioja, especialista en Sociología de la Educación. E-mail: joaquin.giro@unirioja.es
} 


\title{
Resumen
}

La participación de las familias en la escuela es una necesidad dentro del sistema educativo. Su valor es determinante tanto para los resultados escolares como para generar sociedades más democráticas. Sin embargo, en las últimas décadas nos encontramos con una situación paradójica. Por un lado, se valora su importancia y, en teoría, se impulsa. Pero, por otro lado, las legislaciones educativas han influido en una mayor individualización de la participación, y han reducido la importancia de las familias en algunos órganos como el Consejo Escolar. Este artículo analiza la situación de la participación en España, partiendo de la evolución de las legislaciones educativas y contando con los resultados de una amplia investigación cualitativa desarrollada en treinta y un centros de Cataluña, Aragón, Islas Baleares y La Rioja.

Palabras clave: participación; familias; legislación educativa; relación padres-escuela

\begin{abstract}
Family involvement at school is a necessity within the state educational system. Its value is crucial not only for school performance but to promote societies that are more democratic. However, in recent decades we face with a paradoxical situation. On the one hand, we consider its importance and, in theory, this is driven. On the other hand, educational legislation has led to more individualization concerning family involvement, and has reduced the role of families in some school bodies such as the School Board. This paper analyzes the situation of families' involvement in Spain, based on educational legislation development, and counting on the results of an extensive qualitative research carried out in thirty-one centers in Catalonia, Aragon, Balearic Islands and La Rioja.
\end{abstract}

Key Words: involvement; families; educational legislation; family-school relationship 


\section{Introducción}

El Artículo 27 de la Constitución española de 1978 hace referencia al Derecho a la Educación, y dos de sus puntos indican de forma explícita la participación de las familias. El 27.5 asienta la garantía a la Educación a través de la programación general de la enseñanza, la participación efectiva de los agentes implicados, y la creación de centros. Y el 27.7 es todavía más evidente ya que «los profesores, los padres y, en su caso, los alumnos intervendrán en el control y gestión de todos los centros sostenidos por la Administración con fondos públicos en los términos que la ley establezca». Es decir, la participación de las familias en las escuelas y en la Educación se presenta como un hecho consustancial al propio proceso educativo y a su sistema y, lo que es más importante, un derecho. De esta forma, «el reconocimiento del derecho de participación de los padres en el sistema educativo por parte de la Constitución abre la puerta a que los poderes públicos pongan en práctica políticas públicas que faciliten la participación de los ciudadanos en la vida política, económica, cultural y social, tal y como les exige también el constituyente en el artículo 9.2 de la Constitución» (Silveira, 2016: 21).

Por lo tanto, ya la Constitución de 1978 incide en este punto, aunque también genera una dualidad que estará presente en toda la legislación educativa desde entonces, y es el «pacto escolar» entre libertad de elección de centro, institucionalizado por el Artículo 27.3, e igualdad y equidad, imponiéndose a lo largo de estas décadas las visiones que priorizan la primera. Este hecho, junto a otros procesos más generales, determinarán también las políticas educativas y, entre ellas, la participación de las familias en las escuelas (Olmedo, 2008).

Además, la implicación de madres y padres en la escuela también se considera en todos los parámetros de la evaluación de la Educación como un indicador de calidad, desde PISA hasta los elaborados por cualquier otra institución o Administración, adquiriendo una importante dimensión en ese sentido, lo que llevaría a impulsarla y favorecerla (Colás \& Contreras, 2013).

En definitiva, la Educación sería considerada como una corresponsabilidad, superando los tradicionales límites funcionales que asignarían unas tareas claramente definidas, en la que están implicados todos los agentes de la comunidad educativa (estudiantes, madres y padres, docentes, equipos directivos, entorno, Administraciones, etc.). Y tampoco hay que olvidar que numerosos estudios han demostrado la correlación positiva entre la implicación de las madres y padres en la educación de sus hijos/as y los resultados escolares y académicos (Egido, 2015; Reparaz \& Naval, 2014; Bolívar, 2006; Epstein, 2001). Finalmente, el hecho de que las madres y padres participen en las escuelas es una forma también de fomentar sociedades más democráticas, así como de transmitirlas a través del ejemplo para sus hijos e hijas mediante su puesta en práctica (Feito, 2010), lo que daría lugar a que «las razones que encontramos detrás de este reconocimiento jurídico están en primer lugar el de la democracia, en el sentido de que los ciudadanos tienen derecho a participar en los asuntos generales que les conciernen más directamente. $\mathrm{Al}$ respecto, los centros escolares fueron pensados como espacios donde se podría poner en práctica una democracia más cercana a los ciudadanos» (Silveira, 2016: 19).

En las últimas décadas, por lo tanto, la participación de las familias en las escuelas se ha vinculado a nuevas formas de gobernanza, organización de la gestión que pasaría, al 
menos formalmente, por una serie de aspectos que precisan de la incorporación de los agentes de la comunidad educativa, incluidas en un primer plano las familias. Además, este proceso también está relacionado con otra serie de aspectos como el rendimiento de cuentas, la transparencia y con contar con unos canales de comunicación bidireccionales y abiertos entre los diferentes agentes de la comunidad educativa.

La participación de las familias en las escuelas y en la Educación es, por lo tanto, uno de los pilares del sistema educativo, o al menos debería serlo, pero el concepto de participación es amplio y no todo el mundo tiene la misma visión o le otorga iguales significados. Porque esta participación es mucho más compleja y tiene bastantes limitaciones, tanto desde el punto de vista de la institución escolar, como desde las familias, sin dejar de señalar la relativa a las Administraciones. Además, las transformaciones de la sociedad, los cambios acontecidos en las últimas décadas, están afectando no solo a las funciones de las escuelas y las familias, sino también a cómo las segundas valoran a las primeras, a lo que esperan de ellas, a su forma de relacionarse y al propio sistema educativo, complejo y heterogéneo. De lo que no hay duda es que la escuela está asumiendo funciones que antes correspondían a la familia, algunas de ellas vinculadas a la socialización primaria, y lo viene haciendo desde hace décadas, aunque se ha acelerado en los últimos años (Fernández Enguita, 2016; Egido, 2015).

Pero el marco de la participación de las familias en las escuelas parte de los mecanismos habilitados para ello, además de actuaciones proactivas por parte de las Administraciones Públicas, que la posibiliten y la fomenten. Sin embargo, las legislaciones educativas no parecen haberlo logrado exitosamente, especialmente en los aspectos más formalizados e institucionalizados como por ejemplo el Consejo Escolar. Al contrario, las últimas reformas educativas muestran una evolución de los mecanismos de participación de las familias hacia modelos individualizados y donde imperaría una cierta lógica del mercado. Además, se están produciendo desde hace tres décadas dinámicas de privatización que afectan directamente a la participación y a una concepción democrática de la escuela que «están compuestas por la incorporación de un triunvirato: políticas de cuasimercado escolar; dinámicas de gestión de resultados, rendición de cuentas y pago por méritos; y la reconversión de la figura de la dirección escolar desde una perspectiva gestora» (Saura, 2015: 6).

En definitiva, nos encontraríamos ante un «derecho en construcción» (Silveira, 2016), a pesar de todos los avances producidos en estas décadas. Aunque formalmente existe un fomento e incentivación de la participación, además del explícito y necesario reconocimiento de la misma, no es menos cierto que sus resultados no han alcanzado todas sus potencialidades. Al contrario, tanto desde la teoría como desde los trabajos empíricos, se observan las limitaciones, dificultades y barreras que se dan en esa implicación de las familias en las escuelas y en la Educación.

Todo este proceso debe enmarcarse también en un contexto como es el de España, un país que procedía de una dictadura de casi cuatro décadas que dejaría su huella en numerosos aspectos, y uno de ellos es el de la participación de los ciudadanos en la sociedad civil, en sus diferentes ámbitos. Si la transición a la democracia fue un momento reivindicativo, y en el ámbito educativo no se produjo una excepción, las décadas siguientes han visto descender las movilizaciones ciudadanas, salvando momentos puntuales en las dos últimas décadas fundamentalmente. La consecución de parte de las demandas 
tradicionales de parte de las clases trabajadoras, su reducción y la consolidación de las clases medias, la desideologización y despolitización de sectores crecientes de la sociedad, el aumento del conservadurismo y la cada vez mayor presencia de valores neoliberales dieron lugar a una desmovilización social, con buena parte de los ciudadanos asumiendo su rol de votantes cada vez que fuesen convocados. De esta forma, la implicación de las familias en la escuela se ve también determinada por una reducida participación en la vida social y cívica, como lo demuestran los bajos índices de votantes en elecciones a Consejos Escolares que globalmente no suelen superar el 12 \% (Parra et al. 2014; Giró \& Andrés, 2012; Feito, 2007), a elecciones de juntas directivas de Asociaciones de Madres y Padres (AMPAs), o incluso a las dificultades para configurarlas.

Las siguientes páginas recogen la situación de la participación de las familias en la Educación obligatoria, partiendo de una doble perspectiva: por un lado, el análisis de fuentes secundarias sobre la cuestión y, por otro, los resultados derivados de una investigación cualitativa llevada a cabo en centros de Aragón, Cataluña, Islas Baleares y La Rioja. En un primer capítulo, recogemos la evolución legislativa en España en dicha materia, a través de las legislaciones educativas desde la Ley General de Educación de 1970, y es que las políticas educativas también marcan una visión de la participación y del papel que se les quiere dar a las familias en la escuela. En un segundo capítulo, se analizan las formas de implicación, centrándose especialmente en aquellas más institucionalizadas. El tercer apartado aborda la valoración de las mismas a través de la investigación llevada a cabo. Finalmente, en las Conclusiones se trata de vislumbrar la situación de estos mecanismos de participación en un contexto más amplio como es el educativo.

\section{Metodología}

Este artículo se estructura a través de la combinación de materiales teóricos y empíricos. Con respecto a los primeros, se han revisado fuentes secundarias referidas a la participación de las familias en la escuela en España, un objeto de estudio que en los últimos años está alcanzando una especial relevancia tanto por las implicaciones referidas a la comunidad educativa, su generación e interacción entre sus agentes, así como por la demostrada correlación entre la participación y el rendimiento escolar. Además, este proceso ha ocupado siempre una posición transversal en no pocos estudios generales del sistema educativo. También se ha analizado el impacto de las legislaciones en la participación así como las relaciones entre los diferentes agentes implicados, especialmente entre profesorado y familias.

La base del artículo se centra en los resultados de la investigación Familias y escuelas. Discursos y prácticas cotidianas sobre la participación en la educación obligatoria (EDU2012-32657) de la convocatoria 2012 del Subprograma de Proyectos de Investigación Fundamental no Orientada del Ministerio de Economía y Competitividad. Este estudio, desarrollado de 2013 a 2015, se centró en un análisis de experiencias de éxito de la participación de las familias en la escuela en treinta y un centros de Aragón, Cataluña, Islas Baleares y La Rioja. La primera fase del trabajo de campo se fundamentó en entrevistas en profundidad, en total cuarenta y seis, en estas cuatro regiones con informantes cualificados del ámbito de la Educación: políticos, representantes de federaciones y confederaciones de AMPAs, sindicatos educativos, Consejos Escolares 
regionales, movimientos de renovación pedagógica, etc. La segunda fase abordó la realización de etnografías en los centros seleccionados, en función de las variables etapa educativa (Centros de Educación Infantil y Primaria, CEIPs, e Institutos de Educación Secundaria, IES), titularidad del centro (públicos y concertados), ubicación (urbanos y rurales), Comunidades de Aprendizaje y la diversidad del alumnado (centros con elevada concentración de estudiantes de origen extranjero y centros más homogéneos). En todos los centros se entrevistó a los equipos directivos, docentes, madres y padres, AMPAs, personal no docente, orientadores, otros informantes vinculados a los mismos, etc. Igualmente, se realizaron observaciones de las relaciones entre las familias y los centros, se asistió a consejos escolares, reuniones de juntas directivas y asambleas de AMPAs, etc., hasta complementar la etnografía de cada uno de los centros.

\section{La evolución de la legislación educativa y la participación}

El papel de la legislación en la participación de las familias en la escuela es determinante ya que genera y posibilita los cauces y mecanismos para que pueda producirse. En este sentido, la puesta en valor de la participación de las familias en la escuela no debe dejarse de lado de los procesos democratizadores de la sociedad, y la apertura de los centros a las familias no iba a ser una excepción (Egido, 2015).

Pero este proceso no está exento de controversias y polémicas ya que también entran en juego ideologías, visiones de la Educación y del papel que les correspondería a las familias en este sistema, como integrantes de la comunidad educativa. Además, todo este proceso forma parte de un contexto más amplio, el de la propia evolución de la sociedad.

En líneas generales, se puede observar cómo en España el conjunto de las legislaciones educativas han gravitado en gran medida en la dicotomía entre las visiones de la Educación centradas en la libertad de elección de centro y las de la igualdad, dos sensibilidades que están recogidas en la Constitución de 1978 y de difícil encaje. En este sentido, el principal objetivo tras el franquismo era conseguir una educación obligatoria universal, hecho que no se logró hasta el inicio de la década de los ochenta (Feito, 2010).

Con respecto a la participación de las familias en la escuela, el escenario general es que «aunque los pasos han sido en algún momento tímidos y en otros incluso de retroceso, se ha ido reconociendo la necesidad e interés de que los padres estén representados a través de sus asociaciones en el organigrama de la escuela» (Garreta, 2008: 33). Ahora bien, también es cierto que se observa como una especie de evolución en la que «la participación de los padres, entendida como un derecho y una obligación a título colectivo, se ha ampliado a la implicación de las familias a título individual, como medio de mejora de resultados» (Egido, 2015: 16).

En este epígrafe vamos a centrarnos en una exposición de las diferentes legislaciones educativas en relación con la participación, desde el punto de inflexión para el sistema educativo español que supone la Ley General de Educación todavía en pleno franquismo, hasta la más reciente y polémica Ley Orgánica para la Mejora de la Calidad Educativa (LOMCE) de 2013. En estas legislaciones se hace referencia al papel de madres y padres en el sistema educativo, pero también se articulan los mecanismos de participación, su 
peso en los mismos, así como el del resto de los agentes de la comunidad educativa, fundamentalmente los directores de los centros y los docentes, con especial importancia para el Claustro de profesores. En este caso, nos centraremos fundamentalmente en la figura de los Consejos Escolares, por ser la más importante en el ámbito de la toma de decisiones y la que también se ha ido desnaturalizando desde sus orígenes hasta la actualidad.

En la Ley General de Educación (LGE) de 1970 se trata de dar entrada a los actores de la comunidad educativa, incluso se recoge la necesidad de la constitución de asociaciones de madres y padres, pero todo ello desde el tono paternalista del régimen franquista. En definitiva, su puesta en práctica fue muy limitada, y el director del centro se confirmaba como la figura central, quedando el resto de la comunidad educativa sin capacidad de decisión (Garreta, 2008).

Con la Constitución de 1978 reconociendo ese derecho de las familias a participar en la Educación, como hemos visto anteriormente, la Ley Orgánica del Derecho a la Educación (LODE) de 1985, con el Partido Socialista Obrero Español (PSOE) en el poder desde 1982, traería consigo la figura del Consejo Escolar, un intento democratizador de la institución escolar, al menos desde una perspectiva formal (Olmedo, 2008). Este organismo estaba destinado a ser el catalizador de la participación democrática en la escuela de los agentes de la comunidad educativa, con la presencia de todos ellos en el mismo, y como institución decisoria en el centro. Sin embargo, el Consejo Escolar ha devenido en un mecanismo que ha sufrido numerosos cambios con el devenir de las legislaciones educativas, y que no ha desarrollado las potencialidades para los que fue diseñado (Giró \& Andrés, 2012; Vega, 2012; Feito, 2007).

Comenzando con la LODE, los Consejos Escolares tendrían que estar presentes en todos los centros públicos así como los privados que estuviesen financiados por fondos públicos: «al Consejo Escolar del centro se le atribuían importantes competencias, que deberían ser ejercidas de forma participativa, entre las que cabe mencionar: la aprobación del presupuesto del centro, la imposición de sanciones al alumnado, la aprobación del reglamento de régimen interno, las decisiones sobre admisión de alumnos con sujeción a las normas reguladoras existentes, así como la aprobación y evaluación de la programación anual del centro» (Frías del Val, 2014: 73-74).

A pesar de estas premisas, sería el profesorado el que contaría con una mayor representatividad frente al resto de los actores. Además, y paradójicamente, la LODE también supone una transformación, que se iría acelerando, de la figura del director, intensificando sus labores de coordinación y liderazgo. En definitiva, «la LODE no trajo consigo el esperado entusiasmo participativo de la comunidad escolar y muy especialmente de los padres» (Feito, 2010: 58). Pero esta ley será la que ponga las bases de la participación de las familias en la escuela desde 1985 hasta hoy. Siguiendo la Constitución de 1978, madres y padres alcanzan un nuevo estatus en su consideración como actores de primer orden en todo el sistema, además de otorgárseles nuevas potestades a las asociaciones. Pero, en términos generales, la LODE reforzó las competencias de claustros y consejos, incidiendo en el elemento comunitario (Garreta, 2008: 42).

La Ley Orgánica General del Sistema Educativo (LOGSE) de 1990 transformó la Educación en España para adaptarla a los parámetros europeos, así como a los cambios y nuevas necesidades de la sociedad. Los Consejos Escolares tuvieron que adaptarse a 
los nuevos planes de estudios y centros (CEIP e IES), con más presencia de las madres y padres en los primeros, mientras que el Claustro de profesores ganaba protagonismo en los aspectos académicos (Garreta, 2008). En definitiva, los cambios en materia de participación fueron minoritarios. Se incorpora al Consejo Escolar que uno de los representantes de las familias provenga de la Asociación de Madres y Padres más representativa del centro, lo que supone un reconocimiento a este agente colectivo (Frías del Val, 2014).

Sin embargo, la LOGSE es valorada desde diferentes ámbitos como una ley también paradójica ya que irá estableciendo elementos de cuasimercado y, para algunos autores, va a favorecer a las clases medias, no faltando en ella referencias también a la calidad, derivando la responsabilidad educativa a los centros y sus actores, por encima de las Administraciones (Olmedo, 2008). En definitiva, pocos cambios en la participación de las familias en la escuela desde el punto de vista formal, aunque se intuye que la LOGSE contribuirá a poner los cimientos de una participación más individualizada.

La Ley Orgánica de la Participación, la Evaluación y el Gobierno de los Centros Docentes (LOPEG) de 1995 aborda la mejora la participación y la comunicación entre los actores de la comunidad educativa, así como mayores vínculos con el entorno y la realidad social: «se intentó, pues, reforzar la participación en la vida del centro, principalmente en aquellos casos en los que se había observado que presentaba ciertas carencias, como era el sector de los padres y madres de alumnos» (Garreta, 2008: 45). Se apuntala a los Consejos Escolares y el umbral mínimo de representatividad de madres y padres y alumnos en los mismos se sitúa en el 33 \%. Sin embargo, el profesorado no dejaba de ser el mayoritario en este órgano. Así, los cambios que se produjeron tampoco dieron resultado cuando la desmotivación de las familias era creciente. Además, se refuerza la figura del director en aspectos administrativos. De esta forma, «la participación pasa, por tanto, a un segundo plano, tomando un mayor protagonismo los mecanismos de evaluación de la calidad» (Olmedo, 2008: 11).

La LOPEG supuso «un giro hacia prácticas de mayor gestión, responsabilizando a la dirección escolar de la gestión económica. Desde ese momento se elimina en la legislación esa toma de decisiones de los Consejos Escolares, produciendo así, una clara erosión del carácter democrático y participativo de los centros» (Saura, 2015: 8).

La Ley Orgánica de Calidad de la Educación (LOCE) de 2002 fue redactada por el Partido Popular (PP) y apenas llegó a aplicarse. Es una visión de la participación diferente, con la reducción de funciones del Consejo Escolar en la gestión de los centros, siendo un modelo menos participativo, transformando las competencias de los actores educativos. El Consejo Escolar será un órgano consultivo y no interviene, entre otras cuestiones, en la elección del director que con esta nueva ley se realizaría a través de una comisión en la que estarían representados la Administración y el centro. La primacía en la toma de decisiones correspondería a la dirección frente al Consejo Escolar. Se profundiza, por lo tanto, en esa situación en la que «desde la perspectiva de las familias, por el número de representantes que tienen en el Consejo, éstas nunca tuvieron capacidad real para influir, controlar o llevar a cabo propuestas, sino que se encontraban a expensas del claustro y del equipo directivo, siendo, en el mejor de los casos, la llave que determinará el resultado de las disputas de un Claustro dividido» (Olmedo, 2008: 12).

La Ley Orgánica de Educación (LOE) de 2006 trató de corregir algunos aspectos de la LOCE, pero no le devuelve a los consejos la designación o destitución del director del 
centro. Además, el Consejo Escolar no cambiará su composición ni la relación de fuerzas en el mismo, siendo el profesorado el predominante. La LOE intenta recuperar el valor de la participación, reclamando el papel de los Consejos Escolares, pero se produce un retorno a los valores de la LODE, con el profesorado y la dirección manteniendo su peso predominante en estas instituciones, además de no restituir su función en la elección del director o en la resolución de conflictos, tratados estos últimos por los tutores o docentes de forma más rápida.

Finalmente, la Ley Orgánica para la Mejora de la Calidad Educativa (LOMCE) de 2013, es una de las leyes que ha obtenido una mayor contestación por parte de la comunidad educativa (Giró \& Andrés, 2014). Conocida también como «Ley Wert», en referencia a José Ignacio Wert, Ministro de Educación, Cultura y Deporte de 2011 a 2015 e impulsor de la misma, supone la incorporación explícita de modelos neoliberales en la Educación. En relación a la participación de las familias, la LOMCE reduce la misma, incrementando el papel de los directores del centro y reduciendo las atribuciones del Consejo Escolar, que se consolida como un mero órgano consultivo (Giró, 2013).

Tras más de cuatro décadas de evolución de la legislación educativa en España, se puede observar cómo la misma tiene su consecuencia en los derechos y deberes de los agentes de la comunidad educativa: «dichas transformaciones redefinen las funciones y posición de los actores educativos, dotándolos de, o restándoles, nuevos derechos y competencias, generando un nuevo horizonte en el que se espera que desarrollen nuevos roles» (Olmedo, 2008: 3). Como hemos visto, la trayectoria del Consejo Escolar podría ser un indicador del papel de las familias en la Educación, reduciendo su peso en la toma de decisiones, lo que iría en contra de procesos democratizadores y de participación, y contribuiría a que la implicación fuese de carácter más individual que colectiva, como veremos posteriormente.

El otro gran organismo de participación, las AMPAs, se encuentra consolidado y se le considera un agente de primer nivel, aunque también con sus matices y, en no pocos casos, derivado a labores de apoyo en cuestiones más lúdicas. Pero, tanto en el caso de los consejos como de las AMPAs, las legislaciones educativas parecen haber contribuido a la desmotivación en la participación en los mismos. Si ya es complicado movilizar a las familias, el convertirse en meros órganos consultivos, en ser escuchados pero que su opinión no se tenga en cuenta, o encardinarse en unos procesos que vienen de arriba abajo, contribuye a explicar los bajos índices de participación en elecciones a Consejos Escolares, a juntas directivas de las AMPAs, a Asambleas Generales de estas últimas, o a las dificultades para su constitución.

\section{Mecanismos de participación de las familias en la escuela}

No existe una visión unívoca de la participación de las familias en la escuela. Al contrario, en función de los agentes que la definan, el foco de la implicación se centrará en unos aspectos u otros. En todo caso, existe el consenso entre la comunidad educativa en que la participación de madres y padres es necesaria e imprescindible para la consecución del éxito escolar. Además, también se hace referencia a la importancia de los canales de 
comunicación, que se encuentren abiertos y que sean bidireccionales, que no contengan ruidos ni interferencias (Garreta \& Llevot, 2015). Y en este sentido va adquiriendo cada vez más relevancia los relacionados con las Tecnologías de la Información y la Comunicación (TICs) (Macià, 2016).

En definitiva, otros aspectos también quedan evidentes en el valor de la participación de las familias en las escuelas. Por ejemplo, uno de ellos hace referencia a la ya señalada transparencia, es decir, que las familias tengan toda la información posible acerca de lo que ocurre en el centro y afecta a sus hijos. Otro elemento clave para favorecer la participación y la implicación sería la generación de confianza, y para ello son necesarios los canales de comunicación, entre los agentes de la comunidad educativa, especialmente entre familias y docentes. Se parte de que ambos colectivos deben mostrar un mismo mensaje, una misma visión acerca de la educación, ante los hijos/estudiantes, ya que en el caso de que sean disonantes repercutirá negativamente en todo el proceso educativo. Y también la participación supone contar con más información sobre los estudiantes para los docentes, ya que de esta forma podrán realizar mejores diagnósticos sobre su situación y sobre lo que está influyendo en su rendimiento. Sin embargo, también se producen visiones contrapuestas acerca de hasta dónde se debe dar esa implicación de las familias, señalando los profesores y equipos directivos que hay espacios (el aula) y ámbitos (educativo, gestión) exclusivos de ellos (Andrés \& Giró, 2016).

Lo que sí que queda claro en todo el proceso de la participación de las familias en las escuelas es que son los centros, representados por los propios equipos directivos y por los docentes, los que tienen la llave de la misma. Es decir, son estos agentes los que tienen que dar los primeros pasos para la implicación de las madres y padres, para favorecer su entrada en las escuelas, y es que sin partir de esta base no sería posible la participación. El estilo de los equipos directivos y de los profesores, su visión de la implicación de las familias, y las experiencias pasadas en este sentido, marcan cómo se afronta en su conjunto el proceso. Pero también se observa cómo, en la mayor parte de los casos, esa participación se observa desde la perspectiva de seguir las pautas señaladas por la dirección y profesorado, incluso también vinculado a recibir información, ser escuchados y consultados.

En un sentido más amplio, se le otorga un elevado valor y reconocimiento a la generación de una comunidad educativa, de un sentimiento de pertenencia al centro, y de una identidad. La comunidad educativa no puede quedar como un concepto vacío de contenido, al contrario, si no hay un sentimiento de pertenencia difícilmente se puede dar una participación que vaya más allá de los aspectos individuales. Y para la generación de esa comunidad educativa, en la que participan equipos directivos, docentes y familias, pero también estudiantes, el resto del personal de los centros y el propio entorno, es imprescindible profundizar en una concepción más democrática de la implicación de las familias en la escuela.

Resumiendo, y partiendo de los puntos anteriores, se podría observar que hay aspectos comunicativos y colaborativos en la participación de las familias en la escuela, que resumirían dos grandes líneas las formas de implicación (Reparaz \& Naval, 2014). En nuestro planteamiento, descendemos en este sentido en función de dos variables como son el carácter de la misma (formal o informal) y si es colectiva o individual (Giró et al., 2014). 
Con respecto a la participación formal, aquella que está institucionalizada y reglada, destacaría en el lado individual la tutoría. Como veremos en el siguiente epígrafe, es la forma de participación más utilizada por madres y padres. En cuanto a las de carácter colectivo, nos encontraríamos con los ya señalados Consejo Escolar y AMPAs, además de las reuniones colectivas de inicio de curso o trimestrales, en el caso de que se produzcan. Además, también se incluyen en este apartado otras formas de participación reconocidas e institucionalizadas en el Proyecto Educativo de Centro (PEC) o en programaciones como son las fiestas, la formación de padres, o los proyectos, estos últimos muy presentes en la etapa educativa de Infantil.

En la participación informal, aquella que no está institucionalizada o reglada, destacaría en el lado informal los encuentros y conversaciones informales en diferentes espacios (por ejemplo la puerta del centro, la fila a la entrada o salida de clase, el pasillo, incluso la calle, etc.), junto a la petición de información y el apoyo a los deberes y el seguimiento del proceso educativo de los hijos. En el lado colectivo, nos encontraríamos fundamentalmente los movimientos sociales vinculados a la Educación, donde destacarían en los últimos años todos los relacionados con las protestas contra los recortes y la LOMCE («Marea Verde») (Giró \& Andrés, 2014).

¿Cómo han funcionadoy lo hacen en la actualidad estos mecanismos de participación?, ¿qué diferencias existen entre los diferentes niveles educativos?, ¿cómo han afectado las diferentes legislaciones, especialmente en los aspectos más formales, a la implicación de las familias? A estas preguntas respondemos en el siguiente epígrafe.

\section{La valoración del funcionamiento de los mecanismos de participación}

En términos generales, se puede observar que los resultados de las políticas para fomentar la participación han sido limitados y reducidos, no alcanzándose los objetivos propuestos (Egido, 2015; Santa Cruz \& Olmedo, 2011), aunque no hay que olvidar la distancia recorrida y los avances producidos (Colás \& Contreras, 2013). El trabajo de campo desarrollado en nuestra investigación muestra esta visión paradójica, aunque con los matices derivados de la visión que tengan de la participación cada agente interlocutor. Uno de los hechos fundamentales que se demuestran en todo el proceso es cómo la participación desciende a medida que van avanzando los niveles educativos, siendo mayor en Infantil, reduciéndose a lo largo de la Primaria y reduciéndose notablemente en Secundaria, especialmente la de carácter colectivo (Llevot \& Bernard, 2015). El estudio desarrollado también muestra cómo los agentes de la comunidad educativa tienen interiorizadas sus funciones y el rol que ocupan en la Educación, aunque las críticas provienen de buena parte del profesorado (precisamente por no respetar las familias esos límites, según su opinión) y por una parte de las familias (por no poder participar en mayor medida y ámbitos). En su conjunto, existe una desconfianza entre estos dos agentes que determina la participación y lo que esperan los unos de los otros.

Pero la gran mayoría de nuestros entrevistados y entrevistadas asumieron que la participación de las familias va a mejorar el rendimiento escolar y académico de los estudiantes. De esta forma, se demostraría lo que otros autores y autoras han señalado, como 
por ejemplo el identificar a «la colaboración como un medio necesario para incrementar el logro de cada alumno, reducir el fracaso de los grupos desventajados y mejorar los resultados del sistema educativo en su conjunto» (Egido, 2015: 13).

En todo caso, se da la primacía de una participación individual y formal que se refleja en la presencia de la tutoría como el principal mecanismo y el más empleado. Madres y padres se implican en la escuela fundamentalmente a través de estas reuniones individuales, que pueden ser solicitadas por ellos mismos o por los docentes y tutores debido a cuestiones vinculadas con el rendimiento académico o con el comportamiento. Precisamente, una de los lamentos más generalizados de los docentes es que acuden en mayor medida a las tutorías las familias de aquellos estudiantes que menos lo necesitan, y cuyas características socioeconómicas están vinculadas más a las clases medias. Este hecho podría relacionarse con la consideración del modelo de escuela existente, en que la cultura escolar es precisamente la de las clases medias, las más participativas, y para las que la educación de sus hijos puede ser un «proyecto familiar» (Santa Cruz \& Olmedo, 2011), quedando fuera numerosos colectivos que, o bien se sienten excluidos de la institución o bien no cuentan con el capital cultural para implicarse.

Dentro de las tutorías también es necesario resaltar dos cuestiones. Por un lado, cómo a medida que se avanza en las etapas educativas, y especialmente en la Secundaria, las relaciones con los docentes se centran en los tutores, que se convierten en los interlocutores con las familias al especializarse la formación y ampliarse el número de profesores. En segundo lugar, hemos observado una importante disposición por parte de los docentes por adaptarse a los horarios de madres y padres, en numerosas ocasiones incompatibles con los laborales, cambiando las horas de las reuniones.

Es interesante constatar la situación de las reuniones colectivas, en las que participan el conjunto de madres y padres de una clase. Hay que destacar el valor que se otorga desde el conjunto de los actores implicados a las del inicio de curso, en las que se señalan las líneas generales del mismo, los objetivos y las labores a desarrollar. Las familias las valoran positivamente, pero también se produce un descenso de la asistencia de las madres y padres en las trimestrales, en el caso de producirse. Parte del profesorado, especialmente en Secundaria, indican que estas reuniones suelen finalizar con las familias preguntando de forma individual y personal por las cuestiones de sus hijos.

El Consejo Escolar aparece valorado como un órgano importante para la participación de las familias en las escuelas, pero se produce un desencanto con el mismo debido a que las familias están en situación de inferioridad con respecto al centro y los docentes, y las decisiones ya están tomadas (Vallespir et al., 2016). Lo que se debate en el mismo ya está decidido en el Claustro por equipo directivo y profesores. En ese sentido, es normal que madres y padres no se sientan representados y sean conscientes de su rol secundario, descendiendo su participación como se demuestra en las elecciones a Consejo Escolar, como ya apuntaba hace dos décadas Fernández Enguita en La escuela a examen: "de ninguno de los sectores llamados a participar en los órganos de gestión puede decirse que se sienta entusiasmado por la posibilidad ni por sus resultados» (1995: 187).

Como hemos visto en los epígrafes anteriores, la pérdida de funciones del Consejo Escolar es sin duda un retroceso en el papel desempeñado por las familias en la toma de decisiones en la escuela. Este escenario también muestra cómo madres y padres están en una situación asimétrica con respecto a la participación, en una posición de inferioridad 
frente a los profesores. De esta forma, se cuenta con las familias para algunas cuestiones frente a otras, y el concepto de participación se marca «desde arriba».

Un agente fundamental en la participación de carácter colectivo y formal son AMPAs. Convertidas también en un indicador de la implicación de las familias, en nuestra investigación, que se centraba en centros con experiencias de éxito, se pudo comprobar que donde existe un AMPA potente, activa y con madres y padres concienciados, aumenta en sentimiento de comunidad educativa. Sin embargo, las AMPAs también tienen sus limitaciones, la primera de ellas que se basa en un grupo reducido de madres y padres que se implican y forman parte de sus juntas directivas, destinando parte de su tiempo a estas actividades. En segundo lugar, las AMPAs dependen de la renovación de sus integrantes, se dan casos en los que AMPAs muy dinámicas, cuando sus hijos finalizan la escolarización en el centro, pierden parte de su vigencia. Estas situaciones se evidencian en los IES, donde los estudiantes provienen de diferentes CEIPs, y si las AMPAs han sido activas intentan traspasar sus dinámicas al nuevo centro, aunque la participación de las familias es mucho menor. En los centros concertados, la mayoría de los cuales engloban la Educación obligatoria, se observa una mayor continuidad pero no es menos cierto que la participación en Secundaria sigue los mismos parámetros que en los públicos. Finalmente, la asistencia a Asambleas Generales y la participación en elecciones también es reducida, lo que muestra que esa baja motivación que es de carácter general.

Las AMPAs desempeñan un papel muy importante en la generación de una comunidad educativa y como interlocutores con el centro. En relación al primer aspecto, organizan actividades, de carácter formativo, lúdico, etc., que les sitúa en una posición preeminente y visible, apoyándose en no pocas ocasiones los propios centros en ellas para llevarlas a cabo como por ejemplo jornadas culturales, fiestas del colegio, etc. En el segundo aspecto, las AMPAs recogen en numerosas ocasiones propuestas, sugerencias y quejas que trasladan a los equipos directivos y docentes, además de las suyas propias. Pero también las AMPAs señalan que se les escucha pero que parte de sus peticiones no son atendidas. De nuevo, los límites los fijan equipos directivos, docentes y centros en su conjunto.

Las AMPAs, por lo tanto, son un interlocutor y un agente reconocido, cuyas funciones van más allá de la representación de las familias. Por ejemplo, en determinadas comunidades, caso de los centros rurales y en determinados barrios, el AMPA es una más dentro del tejido asociativo, incorporándose e interactuando con el entorno del que forma parte. Además, se suele dar la circunstancia que buena parte de las personas que integran sus juntas directivas también están en otros tipos de movimientos o entidades, como por ejemplo ONGs, asociaciones de vecinos, clubes deportivos, partidos políticos, sindicatos, etc. Por otra parte, las AMPAs contribuyen en los centros aportando ayudas para la realización de actividades, excursiones, viajes, etc., e incluso para aquellas familias que disponen de menos recursos.

Del resto de las formas de participación colectivas y formales, existe una importante diversidad en su éxito. Mientras que las fiestas suelen ser uno de los momentos más importantes en la presencia de las familias en la escuela, la formación de padres se limita a la implicación de un conjunto limitado de madres y padres. Como hemos señalado, en no pocas ocasiones estos proyectos parten de las AMPAs, e incluso también ha habido iniciativas de «Escuelas de Padres» de ayuntamientos y otras entidades, pero de nuevo 
nos encontramos con la falta de tiempo de no pocas madres y padres. Finalmente, el caso de los proyectos es muy ilustrativo en Educación Infantil, el momento de mayor participación colectiva, ya que se invita a las familias a entrar en las aulas e implicarse en los diferentes proyectos de la clase. La edad de los estudiantes, el carácter sobreprotector de madres y padres, y el querer estar presente en todos los momentos posibles de los hijos/ as explicarían en parte para nuestros entrevistados ese éxito en la implicación de las familias en los primeros años de la escolarización, en este caso no obligatoria. Las profesoras y profesores de Infantil buscan y encuentran la colaboración de madres y padres para llevar a cabo parte de sus iniciativas, que generalmente surgen desde los docentes. Sin embargo, cuando se llega a la Educación Primaria, en la mayoría de los centros las familias ya no forman parte de esos proyectos. En esta etapa, los límites y las barreras se fijan más claramente.

Precisamente, a partir de este momento cobran más importancia las cuestiones de seguimiento de los hijos e hijas, así como el apoyo en los deberes. Son mecanismos de participación informales e individuales, y aquí la diversidad de la implicación de las familias es mucho mayor. El otro ámbito de estas características donde también se da una participación de madres y padres es en los encuentros en las entradas y salidas del centro, en el patio, etc. Son situaciones que también tienen sus valoraciones negativas, por ejemplo el entorpecer el discurrir de las filas o el contenido de los mensajes transmitidos, pero en general son bien valorados por las familias porque lo ven como un indicador de cercanía y accesibilidad de los docentes. Sin embargo, algunos equipos directivos son partidarios de limitar estas situaciones. En cuanto a la participación colectiva informal que supondrían los movimientos sociales, hay una diversidad de opiniones en si considerar estos procesos como una forma de participación en la escuela. Los grupos más comprometidos e implicados en los mismos sí que los señalan como tales, además de forma muy activa, aunque no cabe duda que los limitados resultados de la «Marea Verde» y otras movilizaciones los han debilitado.

Existen experiencias que intentan superar las barreras en la participación de las familias en la escuela. Las más explícitas son las Comunidades de Aprendizaje, un modelo que parte precisamente de la implicación y la presencia de los agentes de la comunidad educativa en todos los ámbitos de la escuela, desde la gestión hasta los académicos. Su funcionamiento a través de comisiones, de tertulias dialógicas, de proyectos, etc., los han convertido en referentes de la participación de las familias. Sin embargo, en algunas Comunidades de Aprendizaje analizadas también se observan algunas dificultades que también se dan en el resto de los centros, como es el bajo número de participantes o que siempre sean los mismos.

En definitiva, queda claro que la implicación de las familias en las escuelas sigue siendo una cuestión pendiente (Garreta, 2014, 2007), pero en la que se han producido importantes transformaciones en las dos últimas décadas. Por una parte, el mayor peso corresponde a un modelo más individualizado, que estaría vinculado para algunos autores a las prácticas de cuasimercado en el ámbito de la Educación, vista esta desde la lógica del consumo por lo que «si el centro educativo es considerado como un 'productor' de servicios y las familias como 'consumidoras' de los mismos, definidos ambos de esta forma según el nuevo discurso neoliberal, el ideal de participación y control por parte de las familias sobre el proceso de producción, el proceso educativo en definitiva, no es otro que la elección del 'proveedor' más acorde con los intereses familiares, dejando 
el resto en mano de 'instituciones manufactureras', mediante procesos de ampliación y potenciación de los mecanismos que afiancen la libertad de elección de centro» (Olmedo, 2008: 20). En nuestro trabajo de campo, si bien se observó que esta no es la visión mayoritaria en las familias, sí que está aumentando.

La participación de carácter individual estaría centrada, fundamentalmente, en el rendimiento académico y escolar, así como en preocupaciones por cuestiones disciplinarias, conflictos, etc., especialmente en Secundaria, donde las notas adquieren otro valor en tanto en cuanto determina la trayectoria futura del estudiante. Por otro lado, la participación colectiva, entendida por la preocupación por el conjunto de la clase y de la escuela, habría quedado en un segundo plano, incluso también habiendo desaparecido en gran medida la reivindicación de la participación directa en la escuela, a diferencia de la transición a la democracia y sus primeros años (Giró et al., 2014; Olmedo, 2008). Y no cabe duda de que las legislaciones educativas han contribuido a dotar de menos peso a los órganos de participación institucionalizados, especialmente al Consejo Escolar, en procesos que se retroalimentan y que favorecen también esa individualización. Por otra parte, el carácter de la participación en actividades lúdicas y festivas, más banal y superficial, también es vista desde los agentes más implicados como una implicación de carácter menor y puntual, aunque no es menos cierto que contribuye a crear sentimiento de comunidad.

En todo caso, las relaciones son asimétricas, las partes no están en igualdad de condiciones, y las familias asumirían el rol secundario que se les asigna desde unos programas y actuaciones que vienen determinados desde las escuelas, los equipos directivos, las instituciones, etc. Pero para todos ellos, contar con la colaboración y la implicación de las familias es fundamental, señalan conceptos como la transparencia, el rendimiento de cuentas, los canales de comunicación, etc., pero los cauces los fijan y delimitan ellos, y las posibilidades de las familias de implicarse se centran en los ámbitos ya señalados.

\section{Conclusiones}

La participación de las familias en la escuela sigue estando marcada por la existencia de una «distancia importante entre lo que establecen las leyes y afirman los especialistas en relación al derecho de participación de los padres y madres y la realidad que sucede en los centros escolares» (Silveira, 2016: 24). Incluso, podría señalarse que las diferentes legislaciones educativas, son mucho más participativas formalmente, en su redacción, que en la práctica, siendo aplicación es más restrictiva (Olmedo, 2008; Fernández Enguita, 1995)

Y es que las políticas educativas en relación con la participación, aunque no se pueden disociar del conjunto, también reflejan una visión ideológica, que incide en el papel que se le quiere otorgar a las familias en la escuela, imponiéndose el polo más individual que el colectivo. En consonancia con la evolución de la sociedad, crecen las familias que se comportan en términos de consumo y cuasimercado, a través de la lógica de oferta y demanda, mercantilizando la relación con la escuela. Y algunas reformas educativas han incidido en esta dirección, especialmente la LOMCE. 
En definitiva, nos encontramos con políticas realizadas desde arriba, con unos marcos institucionales que no dejan grandes espacios para las propuestas de las familias o su puesta en marcha. Sí, son escuchadas, pero sus acciones en no pocas ocasiones se limitan a lo que se espera de ellas. Igualmente, existen visiones críticas que inciden en que si figuras como los Consejos Escolares no han tenido éxito o no han calado en las familias es porque no se le ha dado el valor que requiere (Parra et al., 2014).

Además, la participación no es igual en todas las familias, al contrario, son precisamente las de clase media, las más cercanas a la cultura escolar y que se expresan en ese mismo lenguaje, las que más participan. Por el contrario, los colectivos más desfavorecidos, los inmigrantes, las clases trabajadoras, las minorías étnicas, etc., se implican menos cuando es más necesaria su participación, aunque desde algunas perspectivas es la cultura escolar y la institución la que les cierra las puertas, culpabilizándoles a su vez de su baja participación, desidia o incomparecencia (Collet-Sabé et al., 2014). Y es que «la escuela es una institución que beneficia a los grupos ya beneficiados en términos educativos: familias de alto nivel educativo, con muchos libros en casa o pertenecientes a las clases sociales dominantes» (Feito, 2010: 49).

Todo ello en un contexto donde existe una falta de cultura participativa en la sociedad, que en este caso se vería reforzada por las limitaciones y las barreras que se derivan de las legislaciones en materia de la participación de las familias en la escuela. No se produciría un empoderamiento de madres y padres sino que se abocaría por destinarles el papel definido por docentes, directivos, etc. Es decir, se busca la participación y se defiende, pero sin salirse de la senda marcada. Y ante este escenario, pocas son las familias que demandan un nuevo rol. Además, existe una manera de funcionar, de hacer las cosas, que ya está claramente institucionalizada e interiorizada, con la delimitación de funciones por parte de los agentes implicados.

Es necesario insistir en la paradoja que se produce entre la demanda de participación, la queja por la falta de implicación de las familias por parte de los docentes, pero la fijación de la misma a los cauces establecidos. Partimos de la base del peso de las experiencias previas en la visión de la participación, así como del temor existente en ser valorado y evaluado, con buena parte del discurso de los profesores determinado por la desconfianza hacia madres y padres, que es mutua.

Para superar esta gran barrera inicial, es necesaria la formación para la participación, no solo de los docentes, que no están preparados para relacionarse con las familias (apenas existen materias específicas sobre esta cuestión en los planes de estudios de los Grados de Educación Infantil y Primaria, o en los Masters de acceso a la Secundaria), sino también de las propias familias. La formación sería una de las claves en este sentido, produciéndose además dinámicas de retroalimentación entre los distintos agentes de la comunidad educativa (Vigo et al., 2016).

Aunque la responsabilidad es de todos los agentes implicados, son estrategias que deben partir de las Administraciones. Fomentar la formación para mejorar la participación tendría que ser un objetivo fundamental para alcanzar otros fines como por ejemplo la generación de sociedades más democráticas o la lucha contra el fracaso escolar, entre otras. 
Sin embargo, al delimitarse los mecanismos colectivos de participación, o al transformarse su sentido, las relaciones se individualizan y se profundiza en procesos de mercantilización y cuasimercado, quedando lo colectivo en un segundo plano. Este escenario también se enmarcaría en la imposición de tendencias neoliberales en la Educación, siendo las de la LOMCE la máxima expresión, aunque también se observan parte de estos mecanismos en el resto de las legislaciones (Olmedo, 2008; Viñao, 2012; Saura, 2015).

No se han producido movimientos contundentes ni numerosos en contra de la reducción de la participación, de la menor presencia o representatividad de las familias en los órganos formales. A medida que se individualizaba la implicación, o se centraba en aspectos más lúdicos y festivos, se perdía esa visión democrática y colaborativa, ayudado por la legislación que favorecía claramente esa menor participación de las familias en el control y la gestión de los centros. Madres y padres habrían ido perdiendo motivación para participar en los órganos institucionales.

La participación de las familias en la escuela no es una cuestión menor ni complementaria. Al contrario, está demostrado su valor transversal en todo el proceso educativo. Nos encontramos en un escenario en el que las potencialidades de la implicación de madres y padres todavía no se han conseguido, especialmente en el ámbito colectivo, y en este sentido encontraríamos visiones que, desde la comunidad educativa, valoran los avances y logros alcanzados en las últimas décadas, pero otras son más pesimistas, incidiendo en esa individualización, en la desconfianza entre los propios actores implicados, y en la falta de formación para la participación.

\section{Referencias Bibliográficas}

Andrés, S. \& Giró, J. (2016). El papel y la representación del profesorado en la participación de las familias en la escuela. Revista Electrónica Interuniversitaria de Formación del Profesorado, 19 (1), 61-71. Doi: http://dx.doi.org/10.6018/reifop.19.1.245461. Recuperado el 15 de marzo de 2016.

Belda, J.F. (2014). La participación social en la escuela pública: un reto colectivo. Anthropos, 238, 83-102.

Bolívar, A. (2006). Familia y escuela: dos mundos a trabajar en común. Revista de Educación, 339, 119-146. Recuperado el 28 de febrero de 2016 de http://www. mecd.gob.es/revista-de-educacion/numeros-revista-educacion/numerosanteriores/2006/re339/re339_07.html.

Colás, P. \& Contreras, J.A. (2013). La participación de las familias en los centros de Educación Primaria. Revista de Educación Educativa, 31 (2), 485-499. Doi: http:// dx.doi.org/10.6018/rie.31.2.171031. Recuperado el 8 de marzo de 2016.

Collet-Sabé, J., Besalú, X., Feu, J. \& Tort, A. (2014). Escuelas, familias y resultados académicos. Un nuevo modelo de análisis de las relaciones entre docentes y progenitores para el éxito de todo el alumnado. Profesorado. Revista de currículum y formación del profesorado, 18 (2), 7-33. Recuperado el 15 de marzo de 2016 de http://www.ugr.es/ recfpro/rev182ART1.pdf 
Egido, I. (2015). La relaciones entre familia y escuela. Una visión general. Participación Educativa, 7, 11-17. Recuperado el 3 de marzo de 2016 de http://ntic.educacion. es/cee/revista/n7/pdfs/peno7art1_iegido.pdf

Epstein, J.L. (2001). School, Family and Community Partnerships. Preparing Educators and Improving Schools. Boulder: Westview

Feito, R. (2010). Escuela y democracia. Política y Sociedad, 47 (2), 47-61. Recuperado el 17 de marzo de 2016 de https://revistas.ucm.es/index.php/POSO/article/view/ POSO1010230047A

Feito, R. (2007). Balance de la participación de los padres en los Consejos Escolares de centro. Participación Educativa, 4, 4-15. Recuperado el 10 de marzo de 2016 de http://www.mecd.gob.es/revista-cee/pdf/revista-4.pdf

Fernández Enguita, M. (1995). La escuela a examen. Madrid: Pirámide

Fernández Enguita, M. (2016). La Educación en la encrucijada. Madrid: Fundación Santillana

Frías del Val, A.S. (2014). Evolución del marco normativo español sobre la participación de las familias. Contexto histórico. Ministerio de Educación, Cultura y Deporte (Ed.), La participación de las familias en la educación escolar (pp. 57-80). Recuperado el 10 de marzo de 2016 de http://www.mecd.gob.es/dctm/cee/publicaciones/ estudioparticipacion/estudioparticipacion.pdf?documentId=0901e72b81b45e35

Garreta, J. (2014). La participación de las familias en la escuela: una cuestión pendiente. Documentación Social. Revista de Estudios Sociales y Sociología Aplicada, 171, 101-124.

Garreta, J. (2008). La participación de las familias en la escuela pública. Las Asociaciones de Madres y Padres del Alumnado. Madrid: Centro de Investigación y Documentación Educativa (CIDE) y Confederación Española de Asociaciones de Padres y Madres de Alumnos (CEAPA)

Garreta, J. (2007). La relación familia-escuela: ¿una cuestión pendiente? J. Garreta (Ed.), La relación familia-escuela (pp. 9-12). Lleida: Ediciones Universitat de Lleida.

Garreta, J. \& Llevot, N. (2015). Family - school communication in Spain: channels and their use. Ehquidad International Welfare Policies and Social Work Journal, 3, 29-48. Doi: 10.15257/ehquidad.2015.0002. Recuperado el 17 de marzo de 2016.

Giró, J. (2013). Preparando el futuro del sistema educativo con la LOMCE. En Crisis y Cambio: propuestas desde la Sociología. Actas del XI Congreso Español de Sociología, Vol. 2 (pp. 194-204). Madrid: Universidad Complutense de Madrid. Recuperado el 11 de marzo de http://fes-sociologia.com/preparando-el-futurodel-sistema-educativo/congress-papers/1083/

Giró, J. \& Andrés, S. (2014). Las familias se suben a la «Marea Verde». El papel de las madres y padres en las movilizaciones educativas. RASE. Revista de la Asociación de Sociología de la Educación, 7 (3), 627-643. Recuperado el 28 de febrero de 2016 de http://www.ase.es/rase/index.php/RASE/article/view/350 
Giró, J. \& Andrés, S. (2012). Procesos de participación en la Educación y la escuela: el caso de los Consejos Escolares. M. Venegas (Coord.), La Sociología y los retos de la Educación en la España actual. Valencia: Germania.

Giró, J., Mata, A., Vallespir, J. \& Vigo, B. (2014). Familias y escuelas: los diferentes discursos sobrela participación.EhquidadInternational Welfare Policies and Social Work Journal, 2, 65-90. Doi: http://dx.doi.org/10.15257/ehquidad.2014.0009. Recuperado el 15 de marzo de 2016.

Llevot, N. \& Bernard, O. (2015). La participación de las familias en la escuela: factores clave. RASE. Revista de la Asociación de Sociología de la Educación, 8 (1), 57-60. Recuperado el 15 de marzo de 2016 de http://www.ase.es/rase/index.php/RASE/ article/view/370

Macià, M. (2016). La comunicación familia-escuela: el uso de las TIC en los centros de primaria. Revista Electrónica Interuniversitaria de Formación del Profesorado, 19(1), 73-83. DOI: http://dx.doi.org/10.6018/reifop.19.1.245841. Recuperado el 11 de marzo de 2016.

Olmedo, A. (2008). De la Participación Democrática a la Elección de Centro: las Bases del Cuasimercado en la Legislación Educativa Española. Archivos Analíticos de Políticas Educativas, 16 (21). Recuperado el 5 de marzo de 2016 de http://www. redalyc.org/articulo.oa?id=275020545020

Parra, J., García, M.P., Gomariz, M.A. \& Hernández, M.A. (2014). Implicación de las familias en los Consejos Escolares de los centros. Ministerio de Educación, Cultura y Deporte (Ed.), La participación de las familias en la educación escolar (pp. 149-165). Recuperado el 8 de marzo de 2016 de http://www.mecd. gob.es/dctm/cee/publicaciones/estudioparticipacion/estudioparticipacion. pdf?documentId=0901e72b81b45e35

Reparaz, C. \& Naval, C. (2014). Bases conceptuales de la participación de las familias. Ministerio de Educación, Cultura y Deporte (Ed.), La participación de las familias en la educación escolar (pp. 21-34). Recuperado el 4 de marzo de 2016 de http://www.mecd.gob.es/dctm/cee/publicaciones/estudioparticipacion/ estudioparticipacion.pdf?documentId=0901e72b81b45e35

Santa Cruz, E. \& Olmedo, A. (2011). «Sacando lo mejor de cada niño»: la participación de las familias de clase media en el centro y en la educación de sus hijos. Profesorado. Revista de currículum y formación de profesorado, 15 (2), 251-270. Recuperado el 3 de marzo de 2016 de http://www.ugr.es/ recfpro/rev152COL5res.pdf

Saura, G. (2015). Thinks Tanks y educación. Neoliberalismo de FAES en la LOMCE. Archivos Analíticos de Políticas Educativas, 23 (107). Doi: http://dx.doi. org/10.14507/epaa.v23.2106. Recuperado el 10 de marzo de 2016.

Silveira, H. (2016). La participación de las familias en los centros educativos. Un derecho en construcción. Revista Electrónica Interuniversitaria de Formación del Profesorado, 19 (1), 17-29. Doi: http://dx.doi.org/10.6018/reifop.19.1.245511. Recuperado el 7 de marzo de 2016. 
Vallespir, J., Rincón, J.C. \& Morey, M. (2016). La participación de las familias en el Consejo Escolar y la formación del profesorado. Revista Electrónica Interuniversitaria de Formación del Profesorado, 19 (1), 31-45. Doi: http://dx.doi.org/10.6018/ reifop.19.1.245751. Recuperado el 28 de febrero de 2016.

Vega, A. (Coord.) (2012). Indicadores de participación de los padres en la escuela. Un enfoque innovador para una educación de calidad. Madrid: Wolters Kluwer España.

Vigo, B., Dieste, B. \& Thurtson, A. (2016). Aportaciones de un estudio etnográfico sobre la participación de las familias a la formación crítica del profesorado en una escuela inclusiva. Revista Electrónica Interuniversitaria de Formación del Profesorado, 19 (1), 1-14. Doi: http://dx.doi.org/10.6018/reifop.19.1.246341. Recuperado el 15 de marzo de 2016.

Viñao, A. (2012). El desmantelamiento del derecho a la educación: discursos y estrategias neoconservadoras. AREAS. Revista Internacional de Ciencias Sociales, 31, 97107. Recuperado el 27 de febrero de 2016 de http://revistas.um.es/areas/article/ view/165031 